\title{
Randomized controlled trial of supportive-expressive group therapy and body-mind-spirit intervention for Chinese non-metastatic breast cancer patients
}

\author{
Rainbow T. H. Ho ${ }^{1,2}$ - Ted C. T. Fong ${ }^{1}$ - Phyllis H. Y. Lo ${ }^{1}$ - Samuel M. Y. Ho ${ }^{3}$. \\ Peter W. H. Lee ${ }^{4}$ Pamela P. Y. Leung ${ }^{2}$ - David Spiegel ${ }^{5}$ - Cecilia L. W. Chan ${ }^{2}$
}

Received: 7 January 2016 / Accepted: 10 July 2016/Published online: 28 July 2016

(C) The Author(s) 2016. This article is published with open access at Springerlink.com

\begin{abstract}
Purpose This study aimed to evaluate the efficacy of supportive-expressive group (SEG) therapy and body-mindspirit (BMS) intervention on emotional suppression and psychological distress in Chinese breast cancer patients.

Methods This three-arm randomized controlled trial assigned 157 non-metastatic breast cancer patients to BMS, SEG, or social support control group. SEG focused on emotional expression and group support, whereas BMS emphasized relaxation and self-care. All groups received 2-h weekly sessions for 8 weeks. The participants completed measurements on emotional suppression, perceived stress, anxiety, and depression at baseline and three follow-up assessments in 1 year. Results Using latent growth modeling, overall group difference was found for emotional suppression $\left(\chi^{2}(2)=8.88\right.$, $p=0.012)$, marginally for perceived stress $\left(\chi^{2}(2)=5.70\right.$, $p=0.058)$, but not for anxiety and depression $\left(\chi^{2}(2)=0.19\right.$
\end{abstract}

Rainbow T. H. Ho

tinho@hku.hk

Cecilia L. W. Chan

cecichan@hku.hk

1 Centre on Behavioral Health, 2/F, The Hong Kong Jockey Building for Interdisciplinary Research, 5 Sassoon Road, Pokfulam, Hong Kong

2 Department of Social Work \& Social Administration, 5/F, The Jockey Club Tower, The Centennial Campus, The University of Hong Kong, Pokfulam, Hong Kong

3 Department of Applied Social Studies, City University of Hong Kong, Hong Kong, Hong Kong

4 Department of Psychiatry, The University of Hong Kong, Hong Kong, Hong Kong

5 Psychiatry \& Behavioral Sciences, Stanford University, California, USA
$0.94, p>0.05$ ). Post-hoc analyses revealed a significant and moderate reduction (Cohen $d=0.55, p=0.007$ ) in emotional suppression in SEG compared to control group, whereas BMS resulted in a marginally significant and moderate fall $(d=0.46$, $p=0.024)$ in perceived stress. Neither SEG nor BMS significantly improved anxiety and depression $(d<0.20, p>0.05)$. Conclusions The present results did not demonstrate overall effectiveness for either BMS or SEG therapy in the present sample of Chinese non-metastatic breast cancer patients. The participants appear to derive only modest benefits in terms of their psychological well-being from either intervention.

Keywords Breast cancer · Emotional suppression · Body-mind-spirit · Supportive-expressive therapy · Psychological distress $\cdot$ Chinese

\section{Background}

Women with breast cancer experience multiple distressing symptoms such as pain, fatigue, and sleep disturbance [1-3]. The need to undergo a series of arduous radio/chemo-therapy treatments and bear with the cancer-related symptoms often induces psychological distress. A longitudinal study by Lam and colleagues [4] found that initial stress levels of 285 breast cancer patients following cancer diagnosis were predictive of their stress levels 6 years later. Effective expression of negative emotions can help breast cancer patients relieve their distress caused by the traumatic experience [5]. Emotional suppression, defined as deliberate suppression of emotional expression, has been linked with anxiety and depressive symptoms in Western studies on cancer patients [6, 7]. Effective psychological interventions are essential to help improve the psychological well-being of the cancer patients. 
Supportive-expressive group therapy (SEG) is a cognitive, existentially oriented psychotherapy that originated from Western practices [8]. It is an emotion-focused therapy that promotes building social support, emotional expression, and examining existential concerns [9]. Among patients with metastatic breast cancer, SEG has been shown to reduce emotional suppression [9], pain [10, 11], emotional distress [11, 12], and prolong cancer survival [13]. In contrast, the efficacy of SEG in non-metastatic samples appears to be questionable and is relatively unknown. A pilot, single-group study [14] showed improvements in quality of life, cancer-related fatigue, and coping strategies after SEG. Carlson and colleagues [15] found that SEG resulted in more normative diurnal cortisol profiles than control condition in 271 distressed survivors of breast cancer. However, SEG was not found to have significant effects in stress symptoms and quality of life. Similar randomized controlled trials [16-18] did not reveal significant intervention effects for SEG. In the Chinese context, a pilot study [19] supported the feasibility of SEG in that patients were willing and able to talk about personal and negative issues but did not reveal any significant improvements in psychological outcomes.

As opposed to individualism in Western cultures, Chinese culture emphasizes social conformity and collectivism. Chinese people are in general discouraged from expressing negative emotions and concerns for fear of causing further misfortune. They tend to inhibit expression of negative emotions such as anger and anxiety [20] to avoid adding to others' burden. Physical symptoms are plausible signs of somatization that denote suppression of their inner psychological needs. Developed by Chan and colleagues [21], the bodymind-spirit therapy (BMS) is a holistic intervention that addresses the physical body, psychological mind, and spiritual beliefs of the participants and their interconnection. The BMS model integrates Western psychotherapeutic concepts (positive psychology and mindfulness [22]) with Eastern philosophical values (meaning-making and meditation) and Chinese medical practices, such as qigong exercise and acupressure [23]. Under this holistic approach, participants can express their emotions through different channels, including verbal sharing, writing, drawing, singing, and movement. With these non-verbal means of communication, BMS is designed to be culturally sensitive and facilitate patients' expression of their emotions. A recent meta-analysis [24] showed that mindfulness meditation programs had moderate evidence of improved anxiety and depression. Preliminary randomized controlled studies on BMS suggested therapeutic effects for breast cancer patients in terms of reduction in anxiety [25], psychological distress, emotional suppression, and negative mental adjustment [19]. However, a recent study [26] did not find any significant improvements for BMS in depressive symptoms and meaning in life.

Given the evidence base of SEG has predominantly been in Western populations, a systematic evaluation of the efficacy of SEG in a non-Western population is warranted. The present study is novel in evaluating how well SEG generalizes to an Eastern sample of non-metastatic breast cancer patients. A recent study by Carlson and colleagues [15] compared the efficacy of SEG and mindfulness-based intervention for breast cancer survivors, with the latter found to be superior for improving stress levels, quality of life, and social support than the former. To the best of our knowledge, existing studies have yet to compare the efficacy of SEG with BMS on breast cancer patients. The primary objective of the present study was to investigate the effects of the BMS and SEG on emotional suppression and psychological distress, namely, perceived stress, anxiety, and depression in Chinese breast cancer patients. The second objective was to compare the therapeutic effects of the two therapies on the psychological outcomes.

\section{Methods}

\section{Participants and procedures}

The present study adopted a 12-month longitudinal randomized controlled design. This randomized controlled trial was not registered in a clinical trial registry. Participants were recruited from three hospitals and one cancer resource center in Hong Kong between Aug 2002 and May 2004. A total of 258 non-metastatic Chinese breast cancer patients aged between 18 and 65 years old were invited to join the study via invitation mails and follow-up telephone calls. The inclusion criteria were diagnosis of stages I to stage III breast cancer and completion of active treatment. The exclusion criteria included diagnosis of other cancers within the past 10 years, metastasis or recurrence of cancer, major psychiatric illness such as schizophrenia, pregnancy, or the inability to speak or read Chinese.

Potential participants who were interested attended a briefing session during which the study purposes and risks were explained. Written informed consent was obtained from the 157 eligible participants who voluntarily enrolled in the study. Simple randomization with computer generated random numbers (1:1:1 ratio) and allocation concealment were done to randomize participants into the BMS, SEG, or control group. Participants in all groups received 2-h weekly sessions for 8 weeks. Self-report questionnaires on outcome measures were completed at baseline (T0) and three follow-up assessments in the 4th (T1), 8th (T2), and 12th month (T3). Ethical approval was obtained from the local institutional review board of the University of Hong Kong prior to the start of the study.

\section{Interventions}

The BMS therapy was conducted for $2 \mathrm{~h}$ once a week for 8 weeks, with $8-12$ participants per group, and focused on 
nurturing a holistic healthy lifestyle, the normalization of traumatic experiences, and resilience in viewing suffering from cancer as an opportunity for personal growth [19, 21, 27]. It aimed to promote self-acceptance and peace of mind through "letting go" of attachments, searching for meanings of life, and connecting with others through mutual support. A range of activities were offered in the BMS intervention, with each activity lasting around an hour in total for the eight sessions. Examples of physical activities included discussions of a healthy diet, practice of acupressure, breathing and qigong exercises, and guided imagery. Mental well-being activities included discussions of personal growth from the cancer experience, practice of forgiveness and self-love, and promoting awareness of the inner self. Examples of spiritual activities included mindfulness meditation, therapeutic writing, practice of non-attachment and "letting go," and a review of the life journey. Two experienced social workers, who had received standard training in BMS treatment [27], were the group leaders of the sessions.

The SEG therapy comprised weekly 2-h group sessions, with 8-12 participants per group, for 8 weeks, and was based on the supportive-expressive intervention model designed for breast cancer patients [28]. Instead of adopting the full 52week version of SEG intervention, the present study adopted the brief protocol of SEG given the latter is more feasible and practical and matches well with the proposed BMS intervention in terms of total group time $(2 \mathrm{~h} \times 8$ weeks $=16$ total hours). This model focuses on building new bonds of social support, encouraging expression of emotion, re-ordering life priorities, handling the fears of death, fostering interpersonal relationships with family and friends, and improving coping skills. The SEG group was led by two therapists who were a clinical psychologist and a medical social worker, who had received a standardized 2-day training workshop in SEG therapy and had studied the treatment manual. Pilot groups were conducted to allow the therapists practice model implementation and receive feedback by the trainer though no formal assessment was performed on the proficiency in facilitating the pilot groups. The therapists engaged in creating a cohesive and supportive environment. Particular emphases were placed on the expression of depressive feelings, confrontation of fears, fostering new bonds of social support, and acquiring active coping strategies. The therapists received supervision of the SEG group they conducted from the trainer twice at the end of the 1 st and 4 th sessions.

The control group was organized in the form of a social support self-help group and did not include any structured program. It comprised eight weekly sessions that lasted $2 \mathrm{~h}$ each with small groups of 8-12 participants. A social worker provided logistic assistance by recording attendance and time, and preparing tea and snacks. The group members designed the agenda and contents of the session on their own during the scheduled time. Topics such as healthy diet, food, exercises, events during the period, and self-care were discussed in the sessions. The groups shared their worries and showed mutual support for each other.

\section{Measures}

The outcome measures of the present study were emotional suppression, perceived stress, anxiety, and depression. Emotional suppression was assessed by the 21-item, 4-point Chinese Courtauld Emotional Control Scale [20]. This scale measures how often participants suppress feelings of anger, anxiety, or depression, with its total score ranging from 21 to 84. Perceived stress was assessed by the 10-item, 5-point Chinese Perceived Stress Scale [29]. This scale measures how often participants feel stressful in the past month, with its total score ranging from 0 to 40 . Anxiety and depression were measured by the 14-item, 4-point Chinese Hospital Anxiety and Depression Scale [30], with their total scores ranging from 0 to 21 . At baseline, the participants completed the 12-item, binary Chinese General Health Questionnaire [31] as an indicator of baseline psychological functioning with its total score ranging from 0 to 12 . Higher scores denote worse health statuses for all of the measures. In this study, satisfactory reliabilities were found for emotional suppression ( $\alpha=0.80-0.84)$, perceived stress $(\alpha=0.79-0.85)$, anxiety and depression $(\alpha=0.76-0.88)$, and general health $(\alpha=0.90)$. The participants reported demographics characteristics such as age, education level, marital status, income, and cancer duration.

\section{Data analysis}

Analysis of variance and chi-square tests were used to compare the demographic profiles of the groups at baseline. Power analysis for sample size calculation was performed via Monte Carlo simulations [32] on the intervention effects. Under a 4wave longitudinal design with an assumed $20.0 \%$ attrition rate and 0.05 significance level, a total sample size of $n=150$ provided a statistical power of 76.4 and $29.8 \%$ for intervention effects with medium (Cohen $d=0.63$ ) and small effect sizes (Coden $d=0.32$ ), respectively.

Latent growth modeling analyzes the overall trajectories and between-person variation in multiple groups and allows non-linear growth in the variables [33, 34]. The present study examined the intervention effects of BMS and SEG therapies via latent growth modeling in Mplus 7.2 [35] under the robust maximum likelihood estimator. Standard intent-to-treat analyses are allowed using all available data via full information maximum likelihood under the missing-at-random assumption [36]. For each outcome, the 1-year growth trajectories were examined via multi-group unconditional growth models. In the growth models, latent intercept and slope factors denote the baseline status and change over time, respectively. 
Fig. 1 CONSORT flow diagram of participants through the study

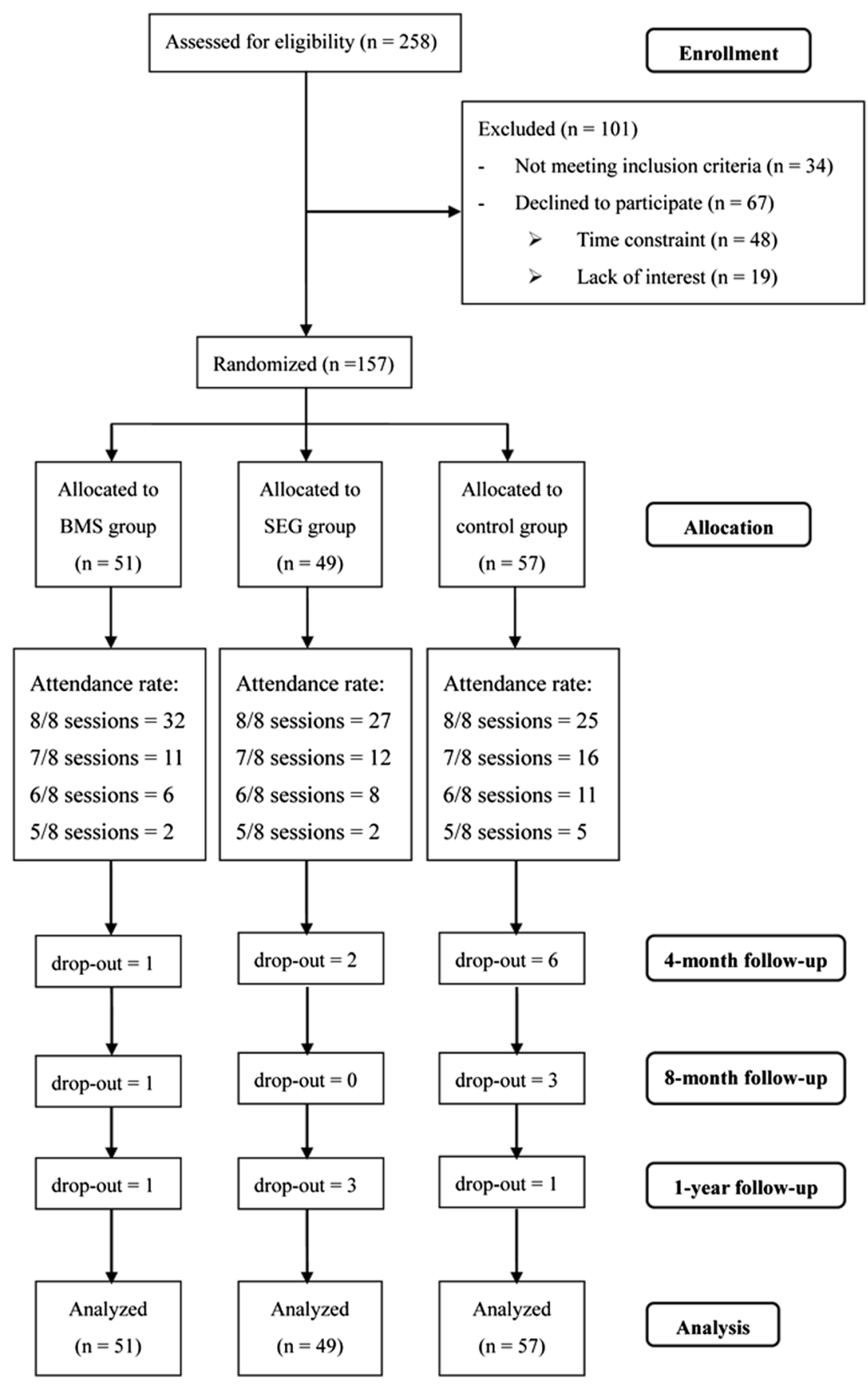

Quadratic growth factors that were statistically significant were added into the models to better fit the trajectory shape. Standardized residuals of the model estimated means were scrutinized in comparison with observed means to detect potential estimation biases. Model fit of the latent growth models was assessed based on the following criteria [37]: insignificant $\chi^{2}(p>0.05)$, comparative fit index $(\mathrm{CFI}) \geq 0.95$, and root mean square error of approximation (RMSEA) $\leq 0.06$.

Overall group difference was analyzed via Wald test of equal slope factors in multi-group conditional growth models, followed by post-hoc evaluations of between-treatment effects over time. Demographic and clinical characteristics that differed significantly across the three groups in preliminary analysis were entered as covariates in the conditional models. In the conditional models, pairwise comparisons were carried out on the slope factors of the outcomes across the three groups. Such comparisons allow us to not only evaluate the effects of BMS and SEG compared to the control group but also compare the intervention effects between BMS and SEG. The overall level of statistical significance was set at $p=0.05$. To control for the multiple comparisons among the three interventions (BMS vs control, SEG vs control, BMS vs SEG), 
post-hoc pairwise comparisons were based on a significance level of $p=0.05 / 3=0.017$ significance level. Effect sizes of the treatment effects were denoted by Cohen $d$ of standardized mean difference, with cut-off of $0.2,0.5$, and 0.8 representing small, moderate, and large magnitudes of difference.

\section{Results}

\section{Participant characteristics}

Figure 1 presents the flow chart of participants through the 12month trial. The 157 participants were randomized into BMS $(n=51)$, SEG $(n=49)$, and control $(n=57)$ group. All of the participants attended at least five out of the eight scheduled sessions. Participants in the BMS, SEG, and control group attended on average 7.4, 7.3, and 7.1 sessions, respectively, suggesting high adherence to treatment protocol on average. Eighteen participants dropped out of the study, implying a 1year drop-out rate of $11.5 \%$. Drop-out rates were similar between the BMS group (5.9 \%) and SEG group (10.2\%) but higher in the control group $(17.5 \%)$. Reasons for dropouts included a lack of time to do the follow-up assessments, failure to contact the participants, returning to work, and refusal to remain in the study. The study completers $(n=139)$ did not differ significantly from the drop-outs $(n=18)$ in terms of baseline characteristics.
Table 1 shows the demographic and baseline characteristics of the participants by group. The participants had a mean age of 47.7 years (standard deviation $(S D)=7.0$ ) with an average cancer duration of 23.4 months ( $\mathrm{SD}=15.1)$, and the majority $(71.3 \%)$ of them were married. The participants showed an acceptable level of general health (mean $=2.6, \mathrm{SD}=3.2$ ) at baseline. The three groups did not differ significantly at baseline except for cancer duration $(\mathrm{F}(2,146)=4.45, p<0.05)$; where the BMS group had a significantly shorter duration than the SEG group. Cancer duration was entered as a control variable in subsequent conditional growth models. Table 2 displays the sample statistics of the outcome variables by group in the four assessment points.

\section{Overall group differences in latent growth models}

The multi-group latent growth models fitted the data well for all outcome variables with insignificant $\chi^{2}$, CFI and TLI $>0.95$, and RMSEA $\leq 0.06$. None of the three groups displayed significant changes $(p>0.05)$ in anxiety and depression. Figures 2 and 3 display the estimated 1-year trajectories of perceived stress and emotional suppression by treatment groups, respectively. For emotional suppression, both BMS and SEG exhibited a concave decreasing trend with significant decreases in the first 4 months and the control group showed no significant change. For perceived stress, neither the SEG nor the control group showed any significant
Table 1 Demographic and clinical characteristics of patients at baseline by group

\begin{tabular}{|c|c|c|c|c|c|}
\hline & $\operatorname{BMS}(N=51)$ & SEG $(N=49)$ & Control $(N=57)$ & & \\
\hline & No. $(\%)$ & No. $(\%)$ & No. $(\%)$ & $\chi^{2}$ & $p$ \\
\hline \multicolumn{6}{|l|}{ Education level } \\
\hline Less than secondary & $15(30)$ & $19(38)$ & $15(26)$ & & \\
\hline Senior secondary & $18(35)$ & $15(31)$ & $27(48)$ & 4.20 & 0.38 \\
\hline Tertiary & $18(35)$ & $15(31)$ & $15(26)$ & & \\
\hline \multicolumn{6}{|l|}{ Marital status } \\
\hline Single & $8(16)$ & $9(18)$ & $14(25)$ & & \\
\hline Married & $37(72)$ & $36(74)$ & $39(68)$ & 1.98 & 0.74 \\
\hline \multirow[t]{2}{*}{ Divorced/separated } & $6(12)$ & $4(8)$ & $4(7)$ & & \\
\hline & Mean (SD) & Mean (SD) & Mean (SD) & $F$ & $p$ \\
\hline Age (years) & $48.8(6.2)$ & $47.2(7.9)$ & $47.2(7.0)$ & 0.85 & 0.43 \\
\hline Cancer duration (months) & $18.6(12.2)$ & $27.2(18.6)$ & $24.8(13.0)$ & 4.45 & $0.01 *$ \\
\hline Income (thousand HKD) & $8.1(11.8)$ & $10.0(15.0)$ & $12.8(15.2)$ & 1.39 & 0.25 \\
\hline General health & $2.3(2.7)$ & $2.7(3.6)$ & $2.8(3.3)$ & 0.39 & 0.68 \\
\hline \multicolumn{6}{|l|}{ Main outcome variable } \\
\hline Perceived stress & $19.2(4.7)$ & $19.2(6.0)$ & $19.0(4.7)$ & 0.03 & 0.97 \\
\hline Anxiety & $6.0(3.4)$ & $6.9(4.2)$ & $7.1(3.5)$ & 1.43 & 0.24 \\
\hline Depression & $4.1(3.0)$ & $4.9(3.6)$ & $4.5(3.0)$ & 0.90 & 0.41 \\
\hline Emotional suppression & $51.7(13.4)$ & $53.6(12.8)$ & $50.9(11.4)$ & 0.88 & 0.42 \\
\hline
\end{tabular}

$S D$ standard deviation, $\chi^{2}$ chi-square value, $F$ F value $* p<0.05$ 
Table 2 Sample statistics of the outcome variables by group in four assessment points

\begin{tabular}{llll}
\hline & BMS & SEG & Control \\
\hline Outcome & M (SD) & M (SD) & M (SD) \\
Perceived stress & & & \\
$\quad$ Time 0 & $19.2(4.7)$ & $19.2(6.0)$ & $19.0(4.7)$ \\
Time 1 & $17.9(4.2)$ & $18.8(6.0)$ & $19.2(5.3)$ \\
Time 2 & $17.4(4.0)$ & $19.0(5.4)$ & $19.2(5.3)$ \\
Time 3 & $17.5(4.1)$ & $18.4(5.5)$ & $19.0(4.8)$ \\
Anxiety & & & \\
Time 0 & $6.0(3.4)$ & $6.9(4.2)$ & $7.1(3.5)$ \\
Time 1 & $5.7(3.5)$ & $7.2(4.5)$ & $6.8(3.3)$ \\
Time 2 & $5.6(3.7)$ & $6.7(4.4)$ & $6.9(3.7)$ \\
Time 3 & $5.7(3.9)$ & $6.6(4.4)$ & $6.6(3.7)$ \\
Depression & & & \\
Time 0 & $4.1(3.0)$ & $4.9(3.6)$ & $4.5(3.0)$ \\
Time 1 & $4.2(2.4)$ & $5.1(4.2)$ & $4.5(3.2)$ \\
Time 2 & $4.2(2.3)$ & $4.3(3.4)$ & $4.6(3.7)$ \\
Time 3 & $4.2(2.7)$ & $5.0(4.3)$ & $4.7(3.6)$ \\
Emotional suppression & & & \\
Time 0 & $51.7(13.4)$ & $53.6(12.8)$ & $50.9(11.4)$ \\
Time 1 & $48.6(10.9)$ & $51.1(12.5)$ & $51.7(10.6)$ \\
Time 2 & $46.8(11.1)$ & $49.5(13.0)$ & $51.2(11.2)$ \\
Time 3 & $48.1(12.9)$ & $50.1(12.4)$ & $52.1(11.5)$ \\
\hline
\end{tabular}

Time 0 = baseline; time $1=4$ month; time $2=8$ month; time $3=12$ month

changes, whereas the BMS group exhibited a significant concave declining trend with significant decreases in the first 4 months. Results of Wald test revealed significant overall group difference for emotional suppression $\left(\chi^{2}(2)=8.88\right.$, $p=0.012)$ but not for anxiety and depression $\left(\chi^{2}(2)=0.19\right.$

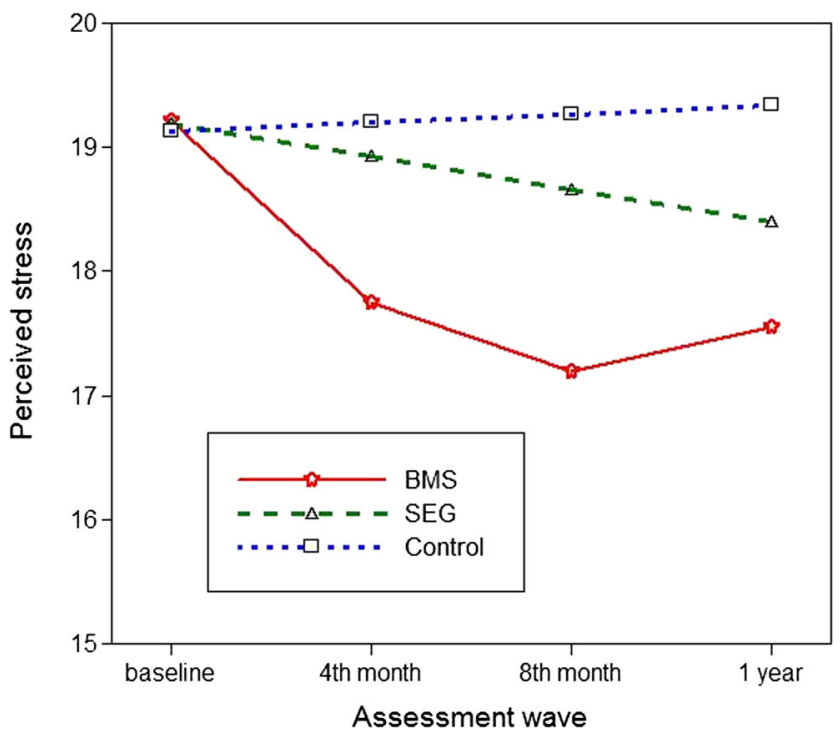

Fig. 2 Model estimated trajectories of perceived stress over 1 year by treatment groups

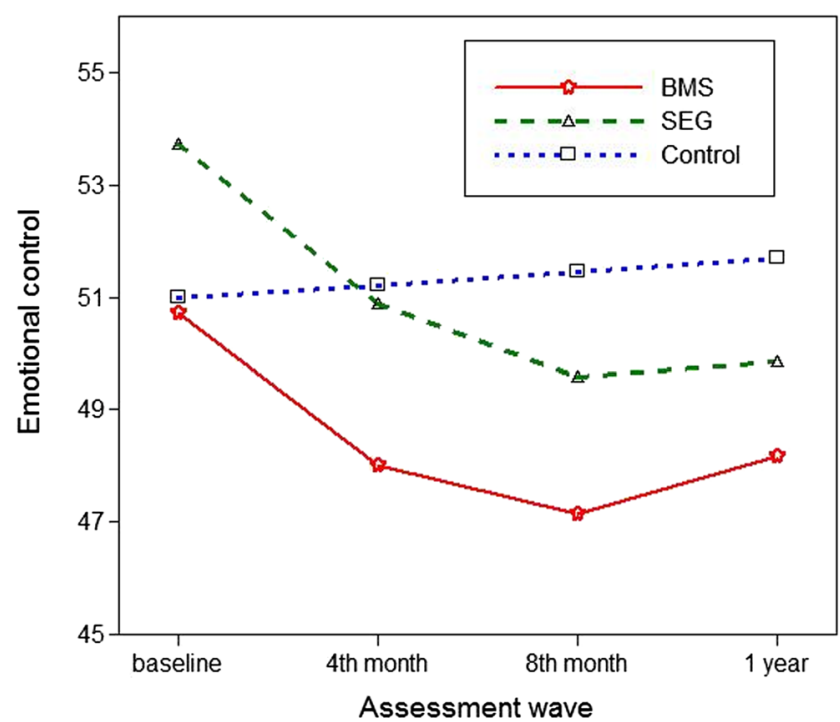

Fig. 3 Model estimated trajectories of emotional suppression over 1 year by treatment groups

$0.94, p>0.05)$. For perceived stress, a marginally significant overall group difference was found $\left(\chi^{2}(2)=5.70, p=0.058\right)$.

\section{Therapeutic effects of BMS and SEG}

Table 3 shows the parameter estimates of BMS and SEG effects on the outcomes in the conditional growth models. Neither SEG nor BMS significantly improved anxiety and depression (Cohen $d<0.20, p>0.05$ ) compared to control group. For emotional suppression, post-hoc analyses revealed a significant and moderate reduction $(d=0.55, p=0.007)$ in SEG compared to control group, while the BMS therapy did not show a significant improvement $(d=0.38, p=0.06)$. For perceived stress, BMS resulted in a marginally significant and moderate fall $(d=0.46, p=0.024)$, while SEG did not show a significant improvement ( $d=0.04, p=0.84$ ) compared to control group. The two interventions produced largely equivalent intervention effects except for the marginally significant decrease $(d=0.42, p=0.037)$ in perceived stress in the BMS group.

\section{Discussion}

This clinical trial investigated the efficacy of BMS and SEG interventions on psychological outcomes in a sample of Chinese patients with non-metastatic breast cancer. The control group showed a stable trend in emotional suppression and psychological distress over the 1-year period. SEG demonstrated significant and moderate decreases $(d=0.55$, $p=0.007$ ) in emotional suppression compared to control group. This therapeutic effect was reflected by a substantial fall in emotional suppression during the first 4 months which 
Table 3 Estimates of the therapeutic effects of BMS and SEG on the outcomes

\begin{tabular}{lllllllllll}
\hline & \multicolumn{2}{l}{ BMS versus control } & \multicolumn{3}{c}{ SEG versus control } & \multicolumn{4}{c}{ BMS versus SEG } \\
\hline Slope & $\mathrm{B}$ & $\mathrm{SE}$ & $\mathrm{d}$ & $\mathrm{B}$ & $\mathrm{SE}$ & $\mathrm{d}$ & $\mathrm{B}$ & $\mathrm{SE}$ & $\mathrm{d}$ \\
Perceived stress & $-1.89^{*}$ & 0.84 & 0.46 & -0.14 & 0.68 & 0.04 & $-1.75^{*}$ & 0.84 & 0.42 \\
Anxiety & -0.14 & 0.44 & 0.07 & 0.00 & 0.43 & 0.00 & -0.15 & 0.37 & 0.08 \\
Depression & 0.32 & 0.33 & 0.20 & 0.24 & 0.39 & 0.12 & 0.09 & 0.33 & 0.05 \\
Emotional suppression & -4.23 & 2.28 & 0.38 & $-5.74^{* *}$ & 2.12 & 0.55 & 1.51 & 2.71 & 0.11 \\
\hline
\end{tabular}

$B$ unstandardized coefficient, $S E$ standard error, $d$ Cohen d effect size

$* * p<0.01 ; * p<0.05$ was well maintained in the following 8 months. Qualitative feedback from the participants revealed perceived benefits such as opportunities to express their feelings, active professional help, and receival of group support. These findings match with results of a 1-year randomized trial [9] where SEG improved the emotion-regulation strategies of women with advanced breast cancer by decreasing their suppression of negative affect without becoming more hostile. This suggests that SEG was applicable in the Chinese context to help non-metastatic cancer patients become more expressive of their emotions.

In contrast, SEG showed minimal effects on perceived stress, anxiety, and depression $(d=0.00-0.12, p>0.05)$ compared to control group. Despite the small sample size, such treatment effects are most likely too small to bear any practical interest regardless of the level of statistical power. The lack of effects for SEG on psychological distress is consistent with previous null findings on psychological adjustment [16], body image [17], quality of life [18], and mood disturbance [38] in patients with metastatic or non-metastatic breast cancer. The present finding may be attributed to the participants' fairly low levels of psychological distress at baseline. In general, studies on psychological interventions that preselected participants according to increased distress showed larger effects [39]. For instance, a pilot study by Grassi and colleagues [40] suggests SEG to be effective in reducing the psychological distress among distressed breast cancer patients with affective disorders. In light of the potential flooring effects, future studies might clarify its intervention effects on psychological distress in more distressed samples.

BMS exerted negligible effects $(d=0.07-0.20)$ on anxiety and depression. This result is similar to a recent randomized trial [26] with null findings for BMS on depressive symptoms and meaning in life. BMS showed small to medium effects $(d=0.38-0.46)$ on emotional suppression and perceived stress. Such effects are consistent with results of a metaanalysis [39] on the effects of psycho-oncologic interventions on emotional distress in cancer patients. Given the current small sample size $(n=157)$, there was only $30 \%$ power to detect a small treatment effect with $d=0.32$ that is statistically significant. The present study showed a lack of adequate power for a precise evaluation of treatment effects of such magnitudes. Indeed, the BMS effect on perceived stress ( $d=0.46, p=0.024$ ) was statistically significant before applying multiple comparison adjustments and could reach statistical significance with a larger sample size. This finding appears to suggest a potential role for BMS in lowering the participants' perceived stress and is consistent with prior research $[39,41]$ that indicates the usefulness of general relaxation interventions in stress-reduction. Moreover, the current intervention leads to sustained benefits in perceived stress throughout the 1-year period, thus potentially demonstrating superior effects to prior relaxation interventions which tend to yield short term effects only [39].

Apart from the small sample size and low statistical power, there are a number of study limitations. First, the self-selection bias in the recruitment of participants casts doubts on the representativeness of the sample. Caution should be taken when generalizing the current findings to other samples of patients with metastatic/non-metastatic breast cancer. Second, the present study only adopted the brief 8-week protocol of SEG intervention rather than the more common 52week intensive version. Given the tendency for longer interventions to produce more sustained effects [39], this difference in treatment dosage could partly explain the overall lack of effectiveness of SEG in the present sample. Third, the present study did not examine treatment adherence as a potential covariate of the intervention effects. Future clinical trials could consider the number of completed sessions as a potential moderator to determine whether people with higher attendance rates benefit more from the intervention. Fourth, the use of self-report measures was subject to a common method bias. Future studies could assess the effects of BMS and SEG on psychophysiological outcome indicators such as salivary cortisol measures. Lastly, we did not assess fidelity of delivery of the models of therapy and cannot conclude whether the general effects of any therapy or the specific components of these interventions explained outcome findings.

The present results did not demonstrate overall effectiveness for either BMS or SEG therapy in the present sample of Chinese non-metastatic breast cancer patients. The participants appear to derive only modest benefits in terms of their psychological well-being from either intervention. No differential treatment effects were found for the two therapies on the 
psychological outcomes. The longitudinal and randomized design and a low 1-year drop-out rate $(11.5 \%)$ lent support to the validity of our findings. The present study suffers from low statistical power given its small sample size and modest treatment effects. Efficacy of the two interventions could be examined in future large-scale studies with adequate statistical power to elucidate the plausible stress-reduction effect of BMS and the role of SEG in lowering emotional suppression among breast cancer patients. Future cross-cultural studies could contrast the intervention effects among patients in the Chinese and Western contexts and examine cultural context as a potential moderator of the intervention effects.

Acknowledgements This study was supported by the Hong Kong Cancer Fund and Hong Kong Research Grants Council (HKU7212/ $03 \mathrm{H}$ ), which did not have any further role in the study design, data collection and analysis, report writing, and decision for paper submission for publication. The authors would like to thank the study participants for their participation and the staff in the three hospitals and the CancerLink of the Hong Kong Cancer Fund for their support in this project.

The authors would like to express their appreciation to Ms. Amy Choi, Ms. Venus Tang, and Mr. Ryder Chan for their help in project coordination and data collection.

Compliance with ethical standards All procedures performed in studies involving human participants were in accordance with the ethical standards of the institutional and/or national research committee and with the 1964 Helsinki declaration and its later amendments or comparable ethical standards. Informed consent was obtained from all individual participants included in the study.

Conflict of interest The authors declare that they have no conflict of interests.

Open Access This article is distributed under the terms of the Creative Commons Attribution-NonCommercial 4.0 International License (http:// creativecommons.org/licenses/by-nc/4.0/), which permits any noncommercial use, distribution, and reproduction in any medium, provided you give appropriate credit to the original author(s) and the source, provide a link to the Creative Commons license, and indicate if changes were made.

\section{References}

1. Ho RTH, Fong TCT, Cheung IKM (2014) Cancer-related fatigue in breast cancer patients: factor mixture models with continuous nonnormal distributions. Qual Life Res 23:2909-2916

2. Bower JE (2008) Behavioral symptoms in patients with breast cancer and survivors. J Clin Oncol 26:768-777

3. Mosher CE, DuHamel KN (2012) An examination of distress, sleep, and fatigue in metastatic breast cancer patients. Psychooncology 21:100-107

4. Lam WWT, Shing YT, Bonanno GA, Mancini AD, Fielding R (2012) Distress trajectories at the first year diagnosis of breast cancer in relation to 6 years survivorship. Psychooncology 21:90-99

5. Watson M, Greer S, Rowden L, Gorman C, Robertson B, Bliss JM, Tunmore R (1991) Relationships between emotional control, adjustment to cancer and depression and anxiety in breast cancer patients. Psychol Med 21:51-57
6. Schlatter MC, Cameron LD (2010) Emotional suppression tendencies as predictors of symptoms, mood, and coping appraisals during AC chemotherapy for breast cancer treatment. Ann Behav Med 40:15-29

7. Iwamitsu Y, Shimoda K, Abe H, Tani T, Okawa M, Buck R (2005) The relation between negative emotional suppression and emotional distress in breast cancer diagnosis and treatment. Health Commun 18:201-215

8. Spiegel D, Classen C (2000) Group therapy for cancer patients: a research based handbook of psychosocial care. Basic Books, New York

9. Giese-Davis J, Koopman C, Butler LD, Classen C, Cordova M, Fobair P, Benson J, Kraemer HC, Spiegel D (2002) Change in emotion-regulation strategy for women with metastatic breast cancer following supportive-expressive group therapy. J Consult Clin Psychol 70:916-925

10. Butler LD, Koopman C, Neri E, Giese-Davis J, Palesh O, ThorneYocam KA, Dimiceli S, Chen X-H, Fobair P, Kraemer HC (2009) Effects of supportive-expressive group therapy on pain in women with metastatic breast cancer. Health Psychol 28:579

11. Goodwin PJ, Leszcz M, Ennis M, Koopmans J, Vincent L, Guther H, Drysdale E, Hundleby M, Chochinov HM, Navarro M, Speca M, Hunter J (2001) The effect of group psychosocial support on survival in metastatic breast cancer. N Engl J Med 345:1719-1726

12. Kissane DW, Grabsch B, Clarke DM, Christie G, Clifton D, Gold S, Hill C, Morgan A, McDermott F, Smith GC (2004) Supportiveexpressive group therapy: the transformation of existential ambivalence into creative living while enhancing adherence to anti-cancer therapies. Psychooncology 13:755-768

13. Giese-Davis J, Collie K, Rancourt KMS, Neri E, Kraemer HC, Spiegel D (2011) Decrease in depression symptoms is associated with longer survival in patients with metastatic breast cancer: a secondary analysis. J Clin Oncol 29:413-420

14. Reuter K, Scholl I, Sillem M, Hasenburg A, Harter M (2010) Implementation and benefits of psychooncological group interventions in German breast centers: a pilot study on supportiveexpressive group therapy for women with primary breast cancer. Breast Care 5:91-96

15. Carlson LE, Doll R, Stephen J, Faris P, Tamagawa R, Drysdale E, Speca M (2013) Randomized controlled trial of mindfulness-based cancer recovery versus supportive expressive group therapy for distressed survivors of breast cancer (MINDSET). J Clin Oncol 31:3119-3126

16. Vos PJ, Garssen B, Visser AP, Duivenvoorden HJ, de Haes HC (2004) Psychosocial intervention for women with primary, nonmetastatic breast cancer: a comparison between participants and non-participants. Psychother Psychosom 73:276-285

17. Vos PJ, Visser AP, Garssen B, Duivenvoorden HJ, Haes HC (2007) Effectiveness of group psychotherapy compared to social support groups in patients with primary, non-metastatic breast cancer. $\mathrm{J}$ Psychosoc Oncol 25:37-60

18. Boesen EH, Karlsen R, Christensen J, Paaschburg B, Nielsen D, Bloch IS, Christiansen B, Jacobsen K, Johansen C (2011) Psychosocial group intervention for patients with primary breast cancer: a randomised trial. Eur J Cancer 47:1363-1372

19. Chan CLW, Ho RTH, Lee PWH, Cheng JYY, Leung PPY, Foo W, Chow LWH, Sham JST, Spiegel D (2006) A randomized controlled trial of psychosocial interventions using the psychophysiological framework for Chinese breast cancer patients. J Psychosoc Oncol 24:3-26

20. Ho RTH, Chan CLW, Ho SMY (2004) Emotional control in Chinese female cancer survivors. Psychooncology 13:808-817

21. Chan CLW, Ho PSY, Chow E (2001) A body-mind-spirit model in health: an Eastern approach. Soc Work Health Care 34:261-282

22. Casellas-Grau A, Font A, Vives J (2014) Positive psychology interventions in breast cancer. A syst Rev Psycho Oncol 23:9-19

23. Ng SM, Fong TCT, Wang XL, Wang YJ (2012) Confirmatory factor analysis of the stagnation scale - a traditional Chinese medicine 
construct operationalized for mental health practice. Int $\mathrm{J}$ Behav Med 19:228-233

24. Goyal M, Singh S, Sibinga EM, Gould NF, Rowland Seymour A, Sharma R, Berger Z, Sleicher D, Maron DD, Shihab HM (2014) Meditation programs for psychological stress and well-being: a systematic review and meta-analysis. JAMA Internal Med 174:357-368

25. Liu CJ, Hsiung PC, Chang KJ, Liu YF, Wang KC, Hsiao FH, Ng SM, Chan CLW (2008) A study on the efficacy of body-mind-spirit group therapy for patients with breast cancer. J Clin Nurs 17:2539-2549

26. Hsiao FH, Jow GM, Kuo WH, Chang KJ, Liu YF, Ho RTH, Ng SM, Chan CLW, Lai YM, Chen YT (2012) The effects of psychotherapy on psychological well-being and diurnal cortisol patterns in breast cancer survivors. Psychother Psychosom 81:173-182

27. Chan CLW (2001) An eastern body-mind-spirit approach: a training manual with one-second techniques. The University of Hong Kong, Hong Kong

28. Classen CC, Diamond S, Soleman A, Fobair P, Spira J, Spiegel D (1993) Brief supportive-expressive group therapy for women with primary breast cancer: a treatment manual. In: Editor (ed)^(eds) Book Brief supportive-expressive group therapy for women with primary breast cancer: a treatment manual. Psychosocial Treatment Laboratory, Stanford, CA

29. Leung DYP, Lam TH, Chan SSC (2010) Three versions of perceived stress scale: validation in a sample of Chinese cardiac patients who smoke. BMC Public Health 10:513

30. Fong TCT, Ho RTH (2013) Factor analyses of the Hospital Anxiety and Depression Scale: a Bayesian structural equation modeling approach. Qual Life Res 22:2857-2863

31. Pan PC, Goldberg D (1990) A comparison of the validity of GHQ12 and CHQ-12 in Chinese primary care patients in Manchester. Psychol Med 20:931-940
32. Muthén LK, Muthén BO (2002) How to use a Monte Carlo study to decide on sample size and determine power. Struct Equ Modeling 9: 599-620

33. Duncan TE, Duncan SC (2004) An introduction to latent growth curve modeling. Behav Ther 35:333-363

34. Curran PJ, Obeidat K, Losardo D (2010) Twelve frequently asked questions about growth curve modeling. J Cogn Dev 11:121-136

35. Muthén LK, Muthén B (1998) Mplus user's guide. Muthen \& Muthen, Los Angeles, CA

36. Little RJA, Rubin DB (1987) Statistical analysis with missing data. Wiley, New York

37. Hu LT, Bentler PM (1999) Cutoff criteria for fit indexes in covariance structure analysis: conventional criteria versus new alternatives. Struct Equ Model 6:1-55

38. Classen C, Butler LD, Koopman C, Miller E, DiMiceli S, Giese Davis J, Fobair P, Carlson RW, Kraemer HC, Spiegel D (2001) Supportive-expressive group therapy and distress in patients with metastatic breast cancer-a randomized clinical intervention trial. Arch Gen Psychiatry 58:494-501

39. Faller H, Schuler M, Richard M, Heckl U, Weis J, Küffner R (2011) Effects of psycho-oncologic interventions on emotional distress and quality of life in adult patients with cancer: systematic review and meta-analysis. J Clin Oncol: JCO 2040:8922

40. Grassi L, Sabato S, Rossi E, Marmai L, Biancosino B (2010) Effects of supportive-expressive group therapy in breast cancer patients with affective disorders: a pilot study. Psychother Psychosom 79:39-47

41. Henderson VP, Clemow L, Massion AO, Hurley TG, Druker S, Hébert JR (2012) The effects of mindfulness-based stress reduction on psychosocial outcomes and quality of life in early-stage breast cancer patients: a randomized trial. Breast Cancer Res Treat 131: 99-109 